\title{
BMJ Open Antenatal melatonin as an antioxidant in human pregnancies complicated by fetal growth restriction - a phase I pilot clinical trial: study protocol
}

To cite: Alers NO, Jenkin G, Miller SL, et al. Antenatal melatonin as an antioxidant in human pregnancies complicated by fetal growth restriction-a phase I pilot clinical trial: study protocol. BMJ Open 2013;3:e004141. doi:10.1136/bmjopen-2013004141

- Prepublication history for this paper is available online. To view these files please visit the journal online (http://dx.doi.org/10.1136/ bmjopen-2013-004141).

Received 27 September 2013 Accepted 20 November 2013

\section{CrossMark}

${ }^{1}$ The Ritchie Centre, Monash Institute of Medical Research, Monash University, Clayton, Victoria, Australia

${ }^{2}$ Department of Obstetrics and Gynaecology, Monash University, Clayton, Victoria, Australia

${ }^{3}$ Monash Women's Services, Monash Health, Clayton, Victoria, Australia

Correspondence to Professor Euan M Wallace; euan.wallace@monash.edu

\section{ABSTRACT}

Background: Fetal growth restriction complicates about $5 \%$ of pregnancies and is commonly caused by placental dysfunction. It is associated with increased risks of perinatal mortality and short-term and long-term morbidity, such as cerebral palsy. Chronic in utero hypoxaemia, inflammation and oxidative stress are likely culprits contributing to the long-term neurological sequelae of fetal growth restriction. In this regard, we propose that melatonin, a powerful antioxidant, might mitigate morbidity and/ or mortality associated with fetal growth restriction. Melatonin has an excellent biosafety profile and crosses the placenta and blood-brain barrier. We present the protocol for a phase I clinical trial to investigate the efficacy of maternal oral melatonin administration in women with a pregnancy complicated by fetal growth restriction.

Methods and analysis: The proposed trial is a single-arm, open-label clinical trial involving 12 women. Severe, early onset fetal growth restriction will be diagnosed by an estimated fetal weight $\leq 10$ th centile in combination with abnormal fetoplacental Doppler studies, occurring before 34 weeks of pregnancy. Baseline measurements of maternal and fetal well-being, levels of oxidative stress and ultrasound and Doppler measurements will be obtained at the time of diagnosis of fetal growth restriction. Women will then start melatonin treatment $(4 \mathrm{mg})$ twice daily until birth. The primary outcomes are the levels of oxidative stress in the maternal and fetal circulation and placenta. Secondary outcomes are fetoplacental Doppler studies (uterine artery, umbilical artery middle cerebral artery and ductus venosus), fetal biometry, fetal biophysical profile and a composite determination of neonatal outcome. A historical cohort of gestational-matched fetal growth restriction and a healthy pregnancy cohort will be used as comparators.

Ethics and dissemination: Ethical approval has been obtained from Monash Health Human Research Ethics Committee B (HREC12133B). Data will be presented at international conferences and published in peer-reviewed journals.

Trial registration number: Clinical Trials, protocol registration system: NCT01695070.
Strengths and limitations of this study

- This pilot study has an appropriate design, and includes sufficient participants, to achieve measureable outcomes.

- Extensive previous human and animal studies form the basis for the design of this trial.

- This pilot study is the first of its kind in the world. Being a pilot study, this trial lacks randomisation and a placebo group, although comparator groups are included. If this trial is successful, the results will be used to inform future randomised controlled trials.

\section{INTRODUCTION}

Fetal growth restriction (FGR) defines the fetus that does not realise its normal growth potential. This significant pregnancy complication accounts for about $5 \%$ of all pregnancies. ${ }^{1}$ FGR is most commonly caused by placental dysfunction. ${ }^{2}$ FGR is strongly associated with increased risks of preterm birth, perinatal death, and, among survivors, significant cardiovascular and neurological morbidities, including cerebral palsy. ${ }^{3-7}$

State-of-the-art fetal surveillance techniques are important in the diagnosis and surveillance of the growth restricted fetus, and afford timely delivery. However, with the exception of antenatal glucocorticoids there are currently no treatments to reduce the short-term and long-term morbidities associated with FGR. ${ }^{8}$ In FGR, injury to the developing fetal brain is likely to principally occur in utero, emphasising that, if the burden of neurological morbidity is to be reduced, then antenatal neuroprotective treatment, as opposed to postnatal therapy, will be necessary. Thus, future treatment should be directed at protecting fetal brain development and reducing the incidence of brain injury before birth rather than solely focusing on care in the nursery. 
Although many in utero events can perturb normal fetal brain development, it is thought that chronic fetoplacental hypoxaemia and oxidative stress are the likely key mechanisms initiating pathways leading to brain injury. ${ }^{9}$ In placental dysfunction, the placenta is exposed to recurrent ischaemia-reperfusion injury, ultimately leading to decreased placental oxygen transfer capacity. Consequently, the placenta cannot adequately meet the growing metabolic needs of the fetus, which in turn becomes progressively hypoxaemic. Compensatory fetal mechanisms attempt to maintain adequate oxygenation, particularly of the heart and brain. Nevertheless, these eventually fail and a complex cascade of metabolic events is initiated that involves the generation of nitrogen and oxygen free radicals. ${ }^{10}$

The central nervous system, and in particular the fetal brain, is particularly susceptible to free radical damage. ${ }^{11}$ Low antioxidant defenses and abundant iron within the fetal brain render it vulnerable to oxidative stress. Furthermore, the fetal brain is rich in membrane lipids that are sensitive to free radical attack. Indeed, lipid peroxidation plays an important role in neuronal and white matter damage directly and indirectly. Byproducts of lipid peroxidation can trigger vasoconstriction, ${ }^{12}$ and are cytotoxic, mutagenic and teratogenic. ${ }^{13-15}$ MRI of preterm infants with white matter injury shows significantly higher levels of protein and lipid peroxidation. ${ }^{16}$ Furthermore, in an autopsy study on human brain tissue, activated microglia, significant protein nitration, lipid peroxidation and cell injury were detected in premyelinating oligodendrocytes in babies diagnosed with periventricular leukomalacia. ${ }^{17}$

Melatonin (5-methoxy- $N$-acetyltryptamine) is an endogenous lipid-soluble hormone, predominantly produced in the pineal gland that is best recognised for its role in providing circadian and seasonal timing cues. It is also a strong antioxidant. It effectively scavenges free radicals, induces endogenous antioxidant enzymes, such as glutathione peroxidase and glutathione reductase ${ }^{18}$ and inhibits the pro-oxidative enzyme nitric oxide synthase. ${ }^{19}$ Melatonin also stabilises cell membranes, enhancing their resistance to oxidative damage. ${ }^{20}$ In vitro, melatonin treatment protects the villous trophoblast against hypoxia-reperfusion-induced oxidative stress and apoptosis. ${ }^{21}$ Owing to its strong antioxidant capacity and its ability to cross the human placenta ${ }^{22}$ and bloodbrain barrier, ${ }^{23}$ melatonin is of particular interest as a candidate antenatal neuroprotectant. The neuroprotective potential of melatonin is augmented by the fact that melatonin inhibits norepinephrine-induced middle cerebral artery constriction ${ }^{24}$ and induces umbilical vasodilation $^{25}$ in ovine models of FGR. In animal models, we have shown that melatonin treatment before and during transient severe fetal asphyxia prevented the formation of hydroxyl radicals within the fetal brain, ${ }^{26}$ reduced lipid peroxidation and cell death and stabilised the blood-brain barrier. ${ }^{27}$ Administration of melatonin at the time of induced hypoxia in the fetal sheep brain also reduced inflammation and cell death. ${ }^{28}$ Using an established ovine model of FGR, ${ }^{29}$ we have now shown that maternal administration of melatonin reduced fetal hypoxia, improved neurodevelopment and decreased brain injury and oxidative stress in newborn lambs (SL Miller, unpublished data 2013).

Melatonin treatment during human pregnancy has been studied for a large range of conditions and at different times during gestation. Predominantly, melatonin has been studied in assisted reproductive technology, aiming to improve oocyte quality and pregnancy rates following in vitro fertilisation (IVF). Melatonin administration, started prior to IVF-cycles and continued during pregnancy, was associated with modestly improved pregnancy outcomes. ${ }^{30-32}$ Importantly, all babies who were born from melatonin-treated pregnancies were born healthy and with no congenital abnormalities (V Unfer, personal communication, 2012). Furthermore, maternal treatment with melatonin significantly boosts placental antioxidant enzyme gene expression. ${ }^{22}$

Except for its known, benign effects on sleep dynamics, melatonin does not exert acute pharmacological effects on the nervous or vascular system. A median lethal dose in mice could not be calculated because an increased mortality rate was not observed, even following administration of extremely high doses of up to $800 \mathrm{mg}$ / $\mathrm{kg}$ melatonin. ${ }^{33}$ Long-term nightly oral treatment with $75 \mathrm{mg}$ melatonin in 1400 non-pregnant women did not cause any serious adverse effects. ${ }^{34}$ To our knowledge, no maternal and/or developmental toxicity effects, due to melatonin treatment, have been reported. The maternal no adverse effect level (NOAEL) and lowest observed adverse effect level (LOAEL) were established at 100 and $200 \mathrm{mg} / \mathrm{kg} /$ day, respectively, for maternally administered melatonin in pregnant rats. The developmental toxicity NOAEL was $\geq 200 \mathrm{mg} / \mathrm{kg} /$ day. ${ }^{35}$

\section{Rationale}

FGR is a serious pregnancy complication, affecting up to $5 \%$ of pregnancies. It is associated with significant fetal and neonatal mortality and morbidity, including neurological impairment. Even though modern obstetrics allow for careful fetal monitoring, there are neither effective therapies for FGR nor established options for antenatal neuroprotection in FGR. Numerous animal studies have established the use of melatonin as an effective antenatal antioxidant and neuroprotectant in acute and chronic hypoxia. In this trial, we aim to explore, for the first time, whether melatonin is an effective antioxidant in human FGR.

\section{Aims}

We will establish the clinical and/or biochemical benefit of maternally administered melatonin as an antioxidant treatment in pregnancies affected by FGR.

Specific aims are:

1. To determine the effect of daily maternal oral melatonin on markers of oxidative stress in the placenta, 
and maternal and fetal circulations in pregnancies affected by the early onset FGR.

2. To determine the effect of daily maternal oral melatonin on the clinical outcomes of pregnancies affected by the early onset FGR.

3. To determine the effect of daily maternal oral melatonin on brain injury and neurodevelopment in growth-restricted newborn babies.

\section{METHODS AND ANALYSIS \\ Study design}

Phase I single-arm, open label clinical trial, with historic cohorts of FGR and healthy pregnancies as comparators.

\section{Subjects}

Twelve women (pregnancies) with FGR will be recruited and studied to completion of the trial. A historical cohort of gestational-matched FGR and a healthy pregnancy cohort will be used as comparators.

\section{Patient eligibility}

Pregnant women with a singleton growth-restricted fetus between $23^{+0}$ and $34^{+0}$ weeks (confirmed gestational age). FGR will be defined as a sonographic-estimated fetal weight at or below the 10th centile for gestational age in combination with at least one abnormal fetoplacental Doppler study of the uterine artery (bilateral uterine artery notching or unilateral uterine artery notching on the ipsilateral side of the placenta), umbilical artery (systolic: diastolic ratio $\geq 95$ th centile or absent, or, reversed end-diastolic flow), middle cerebral artery (pulsatility index $\leq 5$ th centile) or ductus venosus (abnormal A wave and/or pulsatility index $\geq 95$ th centile). For consenting purposes, mothers must be 18 years or older and have a basic understanding of English. Women with an unconfirmed pregnancy duration, a multiple pregnancy or a pregnancy affected by maternal or fetal infection or with a fetus with a chromosomal or major structural anomaly or nonplacental FGR will be excluded from this trial.

\section{Recruitment}

Patients will be recruited from the maternity services of Monash Health and Jessie McPherson Private Hospital, Melbourne, Australia. The study will be staffed with a clinical research team, who will inform potential participants about the aims, methods and potential risks and benefits of this study. Participants will be informed that their participation is voluntary and that they may withdraw consent to participate at any time during the study, and for any reason, and that choosing not to participate or withdraw will not affect their care. An information form about the trial and opportunities to ask questions will be offered to the woman and any other person(s) she chooses. Women will be recruited to the study following written informed consent.
Trial treatment

Following recruitment, and until delivery, women will receive oral melatonin prolonged release (Circadin, Neurim Pharmaceuticals), $4 \mathrm{mg}$ twice daily (a total daily dose of $8 \mathrm{mg}$ ) approximately $12 \mathrm{~h}$ apart. This dose and regimen are based on the results from our own experimental animal research, previously published animal research and clinical trials.

In our experimental FGR study, a maternal, cumulative, intravenous dose of $6 \mathrm{mg} /$ day was administered. The human equivalent dose (HED) was calculated using the FDA-dose-conversion algorithm ${ }^{36}$ and the average Australian height and weight. This resulted in a dose between 7.3 (based on prepregnancy weight) and $8.1 \mathrm{mg} /$ day (based on end-pregnancy weight). As Circadin is only available in $2 \mathrm{mg}$ tablets, the chosen dose of this study is $8 \mathrm{mg} /$ day.

This dose of melatonin is lower than the doses used in several clinical studies in neonates. These trials evaluated melatonin as an antioxidant treatment in newborns with birth asphyxia and other conditions associated with increased oxidative stress. Melatonin was administered orally in doses up to $80 \mathrm{mg} /$ day in these trials without any toxicity. ${ }^{37-41}$ In clinical trials using melatonin to improve assisted reproductive technology, melatonin was administered orally in doses of $3 \mathrm{mg} /$ day before and during pregnancy. ${ }^{30} 32{ }^{42}$ Melatonin treatment, started prior to conception and continued until birth, did not adversely affect pregnancy outcome and fetal or neonatal health (V Unfer, personal communication, 2012). The dose in this trial is higher than previously used doses in human pregnancy but still falls well below the maternal and fetal NOAEL (HED 16 and $32 \mathrm{mg} / \mathrm{kg}$ respectively) and maternal LOAEL (HED $32 \mathrm{mg} / \mathrm{kg}$ ).

Melatonin will be administered orally as $4 \mathrm{mg}$ given twice daily. This dosage regimen is chosen because the terminal half-life of Circadin is $3.5-4 \mathrm{~h}$. In FGR, increased levels of oxidative stress likely occur due to the continuous placental generation of reactive oxygen species. By administering melatonin twice daily, we aim to provide a sustained increase in the maternal and fetal serum. To minimise daytime sleepiness and severe disturbances of circadian rhythms, melatonin will be given early in the morning and at night.

\section{Sample collection}

Prior to starting melatonin, a maternal blood sample will be taken and ultrasound and Doppler measurements will be obtained. This will be repeated once per week until birth. Immediately prior to birth, a maternal blood sample will be obtained. At the time of birth, samples of the amniotic fluid, umbilical cord blood and placenta will be collected. Clinical details of pregnancy, including medication and (multi) vitamin use, delivery and neonatal outcomes will be recorded in prespecified case record forms.

All blood samples will be collected in serum tubes and blood RNA tubes, allowing for measurement of markers 
of oxidative stress and gene expression studies, respectively. Serum tubes will be centrifuged ( $4400 \mathrm{~g}, 15 \mathrm{~min}$ ). The serum will be collected and transferred into collection tubes. The collection tubes will be stored in a $-80^{\circ}$ $\mathrm{C}$ until further processing.

Amniotic fluid will be collected during caesarean section deliveries. The myometrium will be bisected sharply and distinctly from the amnion and chorion. The amniotic fluid will be aspirated using a sterile syringe and 18-gauge drawing-up needle. Samples visibly contaminated with blood will be discarded. Within 30 min of the sample collection, specimens will be transferred to a laboratory. The samples will be centrifuged (1000 g, $15 \mathrm{~min})$ and the supernatant will be collected, transferred into sterile collection tubes and snap frozen. Specimens will be stored at $-80^{\circ} \mathrm{C}$ until further analysis.

Placentas will be obtained at the time of delivery and placed in a sterile tray. The area at the midpoint of the longest distance between the cord insertion and the edge of the placental disc will be sampled. Six full thickness placental samples will be cut out using sterile scissors. The placental tissue samples will be removed from the membranes using sterile forceps and scissors. The placental tissue samples will then be washed in a sterile container using ice cold, sterile phosphate-buffered saline. Two tissue samples will be fixed in $4 \%$ paraformaldehyde and embedded in paraffin for histological analysis. Two tissue samples will be placed in sterile tubes containing RNA later for $24 \mathrm{~h}$ at $4^{\circ} \mathrm{C}$. After immersion in RNA later, these two samples will be transferred into two sterile cryovials, snap frozen in liquid nitrogen and stored at $-80^{\circ} \mathrm{C}$ until further processing for gene expression studies. The remaining tissue samples will be placed into sterile cryovials and snap frozen in liquid nitrogen. Samples will be stored in a $-80^{\circ} \mathrm{C}$ freezer until further processing to measure levels of oxidative stress and melatonin.

\section{Outcome measures}

The primary outcome will be the level of oxidative stress in the placenta and in the maternal (before and during treatment and at delivery) and fetal (umbilical artery and vein at delivery) circulations, as reflected by the measurement of malondialdehyde, 8-isoprostane, total antioxidant capacity and superoxide dismutase. Levels of melatonin will be measured in maternal and fetal blood and in the placenta. Gene expression of pro-oxidant and antioxidant enzymes, melatonin synthesising enzymes, melatonin receptors and clock genes will be measured in the placenta and in the maternal and fetal blood.

The secondary outcome measures will include clinical parameters about the pregnancy and its duration, fetal and neonatal well-being, mortality and morbidity (boxes 1-4).

\section{Follow-up of women and infants}

Participants will be followed weekly for the duration of the trial. The routine clinical care of participants,

\section{Box 1 Ultrasound and Doppler measurements}

Doppler and ultrasound measurements: at recruitment and then weekly until delivery.

Doppler velocimetry: umbilical artery, middle cerebral artery, ductus venosus and maternal uterine arteries

Biometry (fortnightly)

Amniotic fluid index

Fetal behaviour: heart rate, tone, breathing, gross body movements

\section{Box 2 Maternal morbidity}

Markers of maternal morbidity: at recruitment and at least weekly until delivery

Blood pressure

Complete blood count

Liver function tests (aspartate aminotransferase, alanine aminotransferase, $\gamma$-glutamyl transferase and bilirubin)

Renal function tests (urea, creatinine and uric acid)

\section{Box 3 Pregnancy end-points}

\section{Pregnancy outcomes:}

Gestational age at birth

Diagnosis to delivery interval

Abnormal cardiotocogram

Indication for delivery (if not spontaneous)

Mode of birth

Labour analgesia/anaesthesia

Duration of labour stages

Duration of membrane rupture to birth

Used medication such as antihypertensives, magnesium sulfate and corticosteroids

Use of (multi) vitamins and dietary supplements

including ultrasound scans, will not be affected by participation in the study. During the weekly follow-up, fetal and maternal well-being will be assessed and women will discuss any issues that they have experienced. Medication will be supplied weekly to meet the requirements for the following week. Women will be asked to keep a medication and symptom diary (drowsiness,

\section{Box 4 Neonatal outcomes}

Neonatal outcomes:
Sex
Apgar scores (1, 5 and $10 \mathrm{~min})$
Birth weight
Umbilical cord $\mathrm{S100B}$
Head circumference at birth
Composite neonatal outcome: admission to neonatal intensive
care unit, duration of admission, need for and duration of
respiratory support, intraventricular haemorrhage, necrotising
enterocolitis, abnormal neurology, mortality prior to discharge


sleepiness, general physical well-being, time of melatonin intake), which will be reviewed weekly by the principal investigator. Melatonin treatment will cease as soon as birth has occurred. Postnatal clinical care of the women will be the responsibility of the attending obstetric team. The principal investigator will assess the clinical condition of participants daily during the first 3 days postpartum, to ensure that no problems become apparent in response to withdrawal from treatment.

Clinical data will be obtained from neonates who require admission to the neonatal intensive care unit. Long-term follow-up using neurodevelopmental examination and questionnaires and MRI are desirable but will depend on funding.

\section{Sample size and statistical analysis of data}

This study is a proof-of-principle phase I trial. As such, there are no current data available to undertake a formal power calculation to determine the sample size. If successful, this exploratory study will provide the measures needed to perform the power calculations required for the planned phase II randomised controlled trial. This trial will provide data for the establishment of the use of maternal melatonin treatment in FGR.

The results obtained in this trial will be compared to those from historic cohorts of untreated FGR and healthy pregnancies, which we have been collecting since 2011, using the same protocol.

Biomarkers of oxidative stress in the maternal blood samples will be analysed using a repeated measures analysis of variance (ANOVA), including the baseline value as a covariate. It is assumed that the effect of treatment with melatonin on levels of oxidative stress will not depend on duration of treatment. However, secondary analysis will be performed to determine the effect of treatment duration on biomarkers of oxidative stress. If the treatment duration significantly impacts on the effect of melatonin on the oxidative stress status, this will be further investigated using the least-square measure. Data will be plotted as mean scores over time.

Biomarkers of oxidative stress in the maternal blood samples will be analysed using a repeated measures ANOVA, including the baseline value as a covariate. Levels of oxidative stress in the maternal blood sample obtained immediately prior to birth will be compared with samples derived from the historic cohorts using a one-way ANOVA. Levels of oxidative stress and brain injury will be compared with data obtained from the previously described historic cohorts. Clinical data regarding the maternal well-being, pregnancy outcome and neonatal outcome will be compared with the same cohorts. These data will be analysed using a one-way ANOVA.

A $p$ value $\leq 0.05$ is considered to be statistically significant.

\section{Adverse events and safety monitoring}

During the treatment period, clinical care will remain unchanged and will be the responsibility of uninvolved medical staff. The principal investigator will monitor all participants weekly, take a brief medical history, measure blood pressure and interpret ultrasound scan results (obtained by uninvolved staff) to ensure melatonin treatment can be safely continued. A weekly blood sample will be obtained to perform a full blood examination, assess liver and renal function and assess $\mathrm{C}$ reactive protein. For the duration of the trial, the principal investigator will be contactable by telephone at all times.

Any adverse medical events reported by the patient will be recorded in the patients' medical records at each visit. Serious adverse events occurring after initiation of the trial will be reported to the Monash Health Human Research Ethics Committee, Therapeutics Committee and Therapeutic Goods Administration of Australia's Office of Scientific Evaluation within $72 \mathrm{~h}$.

\section{Trial discontinuation or modification}

The participants will be able to end participation at their own request at any time. Participants will be withdrawn from the trial if they or their fetus suffer from an unexpected serious adverse event. Worsening of fetoplacental Doppler velocimetry or fetal growth is within the natural history of FGR and is not part of the discontinuation criteria.

\section{DISCUSSION}

The use of melatonin as an antenatal antioxidant therapy in FGR has been proposed as a promising and safe option to protect the fetal brain from injury. This trial is essential to confirm the antioxidant capacity and safety of melatonin treatment. If this study confirms that maternal melatonin can decrease maternal, fetal and/or placental levels of oxidative stress, we will proceed to a phase II randomised controlled trial to assess the effect of maternal melatonin treatment on brain injury and neurodevelopment. In addition, melatonin treatment could also potentially be used as an antenatal neuroprotectant in other compromised pregnancies or in threatened preterm birth to protect the mother and/or fetus. Finally, if melatonin treatment indeed strengthens antioxidant defenses during pregnancy, it might be a suitable new strategy in the management of pre-eclampsia. A phase I trial to investigate the clinical and biochemical benefit of melatonin treatment in pre-eclampsia has been initiated. ${ }^{43}$

Contributors NOA was involved in the research, contribution of original material, editing and approval of final manuscript. SLM, GJ, EMW were involved in the editing and approval of final manuscript.

Funding This work was supported by the 5Point Foundation.

Competing interests NOA is funded by a Monash Graduate Scholarship and a Monash International Postgraduate Research Scholarship.

Ethics approval This study has been approved by the Monash Health Human Research Ethics Committee B (HREC 12133B)

Provenance and peer review Not commissioned; externally peer reviewed. 
Open Access This is an Open Access article distributed in accordance with the Creative Commons Attribution Non Commercial (CC BY-NC 3.0) license, which permits others to distribute, remix, adapt, build upon this work noncommercially, and license their derivative works on different terms, provided the original work is properly cited and the use is non-commercial. See: http:// creativecommons.org/licenses/by-nc/3.0/

\section{REFERENCES}

1. Robert R. Intrauterine growth restriction. Obstet Gynecol 2002;99:490-6

2. Warkany J, Monroe BB, Sutherland BS. Intrauterine growth retardation. Am J Dis Child 1961;102:249-79.

3. Chase HC. A study of infant mortality from linked records: comparison of neonatal mortality from two cohort studies. United States, January-March 1950 and 1960. Vital Health Stat 20 Data Natl Vital Stat Syst 1972;13:1-99.

4. Ghidini A. Doppler of the ductus venosus in severe preterm fetal growth restriction. Obstet Gynecol 2007;109(2 Pt 1):250-2.

5. Bernstein IM, Horbar JD, Badger GJ, et al. Morbidity and mortality among very-low-birth-weight neonates with intrauterine growth restriction. The Vermont Oxford Network. Am J Obstet Gynecol 2000;182(1 Pt 1):198-206.

6. Ross MG, Beall MH. Adult sequelae of intrauterine growth restriction. Semin Perinatol 2008;32:213-18.

7. Barker DJ. In utero programming of chronic disease. Clin Sci Lond Engl 1979 1998;95:115-28.

8. Miller SL, Wallace EM. Effect of antenatal steroids on haemodynamics in the normally grown and growth restricted fetus. Curr Pediatr Rev 2013;9:67-74.

9. Miller SL, Wallace EM, Walker DW. Antioxidant therapies: a potentia role in perinatal medicine. Neuroendocrinology. 2012;96:13-23.

10. Love S. Oxidative stress in brain ischemia. Brain Pathol Zurich Switz 1999;9:119-31.

11. Halliwell B. Reactive oxygen species and the central nervous system. J Neurochem 1992;59:1609-23.

12. Morrow JD, Minton TA, Roberts LJ II. The F2-isoprostane, 8-epi-prostaglandin F2 $\alpha$, a potent agonist of the vascular thromboxane/endoperoxide receptor, is a platelet thromboxane/ endoperoxide receptor antagonist. Prostaglandins 1992;44:155-63.

13. Schmidt $H$, Grune $T$, Müller $R$, et al. Increased levels of lipid peroxidation products malondialdehyde and 4-hydroxynonenal after perinatal hypoxia. Pediatr Res 1996;40:15-20.

14. Wentzel P, Eriksson UJ. 8-Iso-PGF2 $\alpha$ administration generates dysmorphogenesis and increased lipid peroxidation in rat embryos in vitro. Teratology 2002;66:164-8.

15. Marnett LJ. Lipid peroxidation-DNA damage by malondialdehyde. Mutat Res Mol Mech Mutagen 1999;424:83-95.

16. Inder T, Mocatta T, Darlow B, et al. Elevated free radical products in the cerebrospinal fluid of VLBW infants with cerebral white matter injury. Pediatr Res 2002;52:213-18.

17. Haynes RL, Folkerth RD, Keefe RJ, et al. Nitrosative and oxidative injury to premyelinating oligodendrocytes in periventricular leukomalacia. J Neuropathol Exp Neurol 2003;62:441-50.

18. Pablos MI, Chuang J, Reiter RJ, et al. Time course of the melatonin-induced increase in glutathione peroxidase activity in chick tissues. Biol Signals 1995;4:325-30.

19. Pozo D, Reiter RJ, Calvo JR, et al. Inhibition of cerebellar nitric oxide synthase and cyclic GMP production by melatonin via complex formation with calmodulin. J Cell Biochem 1997;65:430-42.

20. García JJ, Reiter RJ, Guerrero JM, et al. Melatonin prevents changes in microsomal membrane fluidity during induced lipid peroxidation. FEBS Lett 1997;408:297-300.

21. Lanoix D, Lacasse A-A, Reiter RJ, et al. Melatonin: The watchdog of villous trophoblast homeostasis against hypoxia/reoxygenationinduced oxidative stress and apoptosis. Mol Cell Endocrinol 2013;381:35-45.
22. Okatani Y, Okamoto K, Hayashi K, et al. Maternal-fetal transfer of melatonin in pregnant women near term. J Pineal Res 1998;25: 129-34.

23. Okatani $\mathrm{Y}$, Wakatsuki $\mathrm{A}$, Kaneda C. Melatonin increases activities of glutathione peroxidase and superoxide dismutase in fetal rat brain. J Pineal Res 2000;28:89-96.

24. Torres-Farfan C, Valenzuela FJ, Mondaca M, et al. Evidence of a role for melatonin in fetal sheep physiology: direct actions of melatonin on fetal cerebral artery, brown adipose tissue and adrenal gland. J Physiol 2008;586:4017-27.

25. Thakor AS, Herrera EA, Serón Ferré $M$, et al. Melatonin and vitamin $C$ increase umbilical blood flow via nitric oxide dependent mechanisms. J Pineal Res 2010;49:399-406.

26. Miller SL, Yan EB, Castillo-Melendez M, et al. Melatonin provides neuroprotection in the late-gestation fetal sheep brain in response to umbilical cord occlusion. Dev Neurosci 2005;27:200-10.

27. Yawno T, Castillo-Melendez M, Jenkin G, et al. Mechanisms of melatonin-induced protection in the brain of late gestation fetal sheep in response to hypoxia. Dev Neurosci 2012;34:543-51.

28. Welin A-K, Svedin P, Lapatto $\mathrm{R}$, et al. Melatonin reduces inflammation and cell death in white matter in the mid-gestation fetal sheep following umbilical cord occlusion. Pediatr Res 2007;61:153-8.

29. Supramaniam VG, Jenkin G, Loose J, et al. Chronic fetal hypoxia increases activin A concentrations in the late-pregnant sheep. BJOG Int J Obstet Gynaecol 2006;113:102-9.

30. Unfer V, Raffone E, Rizzo P, et al. Effect of a supplementation with myo-inositol plus melatonin on oocyte quality in women who failed to conceive in previous in vitro fertilization cycles for poor oocyte quality: a prospective, longitudinal, cohort study. Gynecol Endocrinol 2011;27:857-61.

31. Tamura $\mathrm{H}$, Nakamura $\mathrm{Y}$, Terron MP, et al. Melatonin and pregnancy in the human. Reprod Toxicol 2008;25:291-303.

32. Benedetto PPRERV. Effect of the treatment with myo-inositol plus folic acid plus melatonin in comparison with a treatment with myo-inositol plus folic acid on oocyte quality and pregnancy outcome in IVF cycles. A prospective, clinical trial. Eur Rev Med Pharmacol Sci 2010;14:555.

33. Barchas J, Dacosta F, Spector S. Acute pharmacology of melatonin. Nature 1967;214:919-20.

34. Silman RE. Melatonin: a contraceptive for the nineties. Eur J Obstet Gynecol Reprod Biol 1993;49:3-9.

35. Jahnke G, Marr M, Myers C, et al. Maternal and developmental toxicity evaluation of melatonin administered orally to pregnant Sprague-Dawley rats. Toxicol Sci Off J Soc Toxicol 1999;50:271-9.

36. U.S. Food and Drug Administration, Guidance for Industry Estimating the Maximum Safe Starting Dose in Initial Clinical Trials for Therapeutics in Adult Healthy Volunteers, Pharmacology and Toxicology, 2005, http://www.fda.gov/cder/guidance/index.htm

37. Gitto E, Reiter RJ, Cordaro SP, et al. Oxidative and inflammatory parameters in respiratory distress syndrome of preterm newborns: beneficial effects of melatonin. Am J Perinatol 2004;21:209-16.

38. Gitto E, Romeo C, Reiter RJ, et al. Melatonin reduces oxidative stress in surgical neonates. J Pediatr Surg 2004;39:184-9; discussion 184-9.

39. Gitto E, Karbownik M, Reiter RJ, et al. Effects of melatonin treatment in septic newborns. Pediatr Res 2001;50:756.

40. Fulia F, Gitto E, Cuzzocrea S, et al. Increased levels of malondialdehyde and nitrite/nitrate in the blood of asphyxiated newborns: reduction by melatonin. J Pineal Res 2001;31:343-9.

41. Gitto E, Aversa S, Salpietro CD, et al. Pain in neonatal intensive care: role of melatonin as an analgesic antioxidant. $J$ Pineal Res.2012;52:291-5.

42. Tamura H, Takasaki A, Miwa I, et al. Oxidative stress impairs oocyte quality and melatonin protects oocytes from free radical damage and improves fertilization rate. J Pineal Res 2008;44:280-7.

43. Hobson SR, Lim R, Gardiner EE, et al. Phase I pilot clinical trial of antenatal maternally administered melatonin to decrease the level of oxidative stress in human pregnancies affected by pre-eclampsia (PAMPR): study protocol. BMJ Open 2013;3:e003788. 